\title{
CRESCIMENTO DA ACÁCIA-NEGRA EM RESPOSTA A APLICAÇÃO DE NITROGÊNIO, FÓSFORO E POTÁSSIO
}

\author{
Mauro Valdir Schumacher ${ }^{1}$, Márcio Viera ${ }^{2}$, Eduardo Kneipp Londero ${ }^{3}$, \\ Francine Neves Calili ${ }^{4}$, Vicente Guilherme Lopes ${ }^{5}$, Rudi Witschoreck ${ }^{6}$
}

(recebido: 3 de agosto de 2010; aceito: 28 de setembro de 2012)

\begin{abstract}
RESUMO: Em razão da escassez de informações sobre adubação em acácia-negra, no presente estudo, objetivou-se avaliar o crescimento de plantas de acácia-negra em função de diferentes níveis de adubação com nitrogênio, fósforo e potássio, após seis anos da implantação. O delineamento utilizado foi de blocos ao acaso, com distribuição trifatorial (doses de fertilizantes). Avaliou-se a altura total $(\mathrm{m})$, diâmetro a altura do peito (DAP) $(\mathrm{cm})$ e volume de madeira com casca $\left(\mathrm{m}^{3} \mathrm{ha}^{-1}\right)$. A acácia-negra apresentou resposta positiva e significativa, de crescimento às adubações com $\mathrm{N}$ e $\mathrm{P}$ (interação) e ausência de resposta ao K. Para a obtenção do máximo crescimento da acácia-negra, para a condição edafoclimática estudada, deve-se utilizar a dose máxima de nitrogênio $\left(40,0 \mathrm{~kg} \mathrm{ha}{ }^{-1} \mathrm{de} \mathrm{N}\right)$ e 78,9 $\mathrm{kg} \mathrm{ha}^{-1}$ de fósforo, não sendo necessária a adição de potássio via fertilizantes.
\end{abstract}

Palavras-chave: Acacia mearnsii, nutrição florestal, adubação.

\section{BLACK-WATTLE GROWTH IN REPONSE TO APPLICATION OF NITROGEN, PHOSPHORUS AND POTASSIUM}

\begin{abstract}
Due to the lack of information about Black-wattle fertilization, this study evaluated black-wattle plants growth in response to different fertilization levels of nitrogen, phosphorus and potassium six years after implantation. The statistical design used was a randomized blocks with trifatorial distribution. Total height $(\mathrm{m})$, diameter at breast height $(\mathrm{DBH})(\mathrm{cm})$ and stem volume with bark $\left(\mathrm{m}^{3} \mathrm{ha} \mathrm{a}^{-1}\right)$ were evaluated. Black-wattle showed a positive and significant growth response to $N$ and $P$ (interaction) fertilizations and absence for $K$. $m$ To obtain the maximum development of black-wattle, for the soil and climate condition studied, it is required the

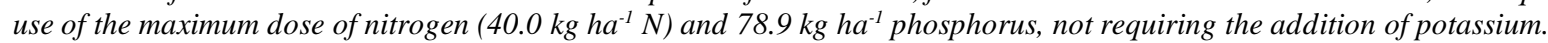

Key words: Acacia mearnsii, forestry nutrition, fertilization.

\section{INTRODUÇÃO}

A Acacia mearnsii De Wild., conhecida como acácia-negra, é originária do leste da Austrália, desde a latitude $33^{\circ} 43^{\prime} \mathrm{S}$ (Balburra) até $42^{\circ}$ 58' (Hobart, Tasmânia). Ocorre naturalmente desde o nível do mar até altitudes superiores a $1.000 \mathrm{~m}$ na região de Cooma. A precipitação na sua região de ocorrência é, em geral, menor que 1.000 $\mathrm{mm}$ anuais, e nas regiões mais frias (Cooma, Monaro, Tableland, NSW), chega a ocorrer oitenta geadas por ano, com mínimas absolutas entre $-10^{\circ} \mathrm{Ce}-12^{\circ} \mathrm{C}$ (SIMON, 2005). São árvores monopodiais, de caule mais ou menos reto na maior parte da altura e possuem folhagem verde-escura, folhas perenes, compostas e bipinadas (SCHÖNAU, 1969). No Brasil, é cultivada quase que na totalidade no Estado do Rio Grande do Sul, onde ela foi introduzida em 1928, em conjunto com atividade agrícola e pecuária. Estima-se que o plantio atual no estado esteja em torno de 130 mil hectares, envolvendo cerca de 40 mil famílias de pequenos produtores rurais (MOCHIUTTI, 2007; SIMON, 2005).

Os plantios dessa espécie normalmente são realizados em solos com baixo nível de fertilidade natural e, muitas vezes, as práticas de manejo do solo não são realizadas corretamente, diminuindo o potencial produtivo

${ }^{1}$ Engenheiro Florestal, Professor Doutor em Ecologia e Nutrição Florestal - Universidade Federal de Santa Maria - Centro de Ciências Rurais - Departamento de Ciências Florestais - 97105-900 - Santa Maria, RS, Brasil - mvschumacher@gmail.com

${ }^{2}$ Engenheiro Florestal, Professor Doutor em Engenharia Florestal - Universidade Federal de Santa Maria - Unidade Descentralizada de Educação Superior de Silveira Martins - Rua Francisco Guerino, 407, Centro - 97195-000 - Silveira Martins, RS, Brasil - vieraflorestal@yahoo.com.br ${ }^{3}$ Engenheiro Florestal, Mestre em Engenharia Florestal - Rua Vicente do Prado Lima, 554/203 - Camobi - 97105390 - Santa Maria, RS, Brasil - eklondero@gmail.com

${ }^{4}$ Engenheira Florestal, Professora Doutora em Engenharia Florestal - Universidade Federal de Goiás - Escola de Agronomia - Rodovia Goiânia - Nova Veneza, km 0 - Campus Samambaia - Cx. P. 131 - 74001-970 - Goiania, GO, Brasil - francine.calil@terra.com.br ${ }^{5}$ Engenheiro Florestal, Doutorando em Engenharia Florestal - Universidade Federal de Santa Maria - Centro de Ciências Rurais Departamento de Ciências Florestais - 97105-900 - Santa Maria, RS, Brasil - viglopes@yahoo.com.br

${ }^{6}$ Engenheiro Florestal, Mestre em Engenharia Florestal - Universidade Federal de Santa Maria - Centro de Ciências Rurais - Departamento de Ciências Florestais - Laboratório de Ecologia Florestal - 97105-900 - Santa Maria, RS, Brasil - r.witschoreck@yahoo.com.br 
do povoamento. Embora plantas adaptadas a baixa disponibilidade de nutrientes possam se desenvolver (PARDOS et al., 2005), é indispensável a adoção de práticas de manejo e correção da fertilidade do solo em áreas cultivadas com acácia-negra, a fim de elevar a produtividade dos sítios florestais (DALLAGO, 2000; PINKARD, 2003; SMETHURST, 2000), ou pelo menos mantê-la para rotações futuras.

Essas práticas de manejo devem levar em consideração a adoção de fontes de nutrientes por meio da adubação mineral ou orgânica (GONÇALVES, 1995; VOGEL, 2002), fazendo com que o solo forneça todos os nutrientes em quantidades necessárias para a obtenção do crescimento desejado para as plantas (FORRESTER et al., 2006). Existem poucos estudos sobre o efeito obtido com a adição de fertilizantes em essências florestais (GONÇALVES; BARROS, 1999), e que as características e quantidade de adubos a serem aplicados dependerão das necessidades nutricionais da espécie utilizada, da fertilidade do solo, da forma de reação dos adubos com o solo, da eficiência dos adubos, de fatores de ordem econômica (GONÇALVES, 1995; GONÇALVES; BARROS, 1999; SMETHURST, 2010) e da disponibilidade hídrica (PARDOS et al., 2005; RYAN, 2010). Portanto, para a nutrição adequada das árvores, deve-se balancear a sua demanda com a oferta de nutrientes, no tempo e no espaço (BARROS; NOVAIS, 1990).

A adubação florestal, com raras exceções, é realizada de modo empírico e utilizando-se apenas uma única formulação de nitrogênio, fósforo e potássio, independente do tipo de solo, da espécie e da época de plantio (BARROS; NOVAIS, 1990). Essa exceção se aplicada às diferentes espécies e materiais genéticos de eucaliptos, os quais recebem de duas a três aplicações de fertilizantes durante o seu ciclo de 6 a 7 anos (BARROS et al., 2004; GONÇALVES et al., 2008). A adição de fertilizantes deveria ser, pelo menos, igual à removida do sítio pela colheita florestal para que a produtividade seja mantida (CALDEIRA et al., 1999, 2003).
Em decorrência disso, fundamenta-se a importância da realização de estudos envolvendo diferentes doses de adubação para a Acacia mearnsii, visando à obtenção de maior produtividade e sustentabilidade na produção florestal, por meio da recomendação adequada da dose de NPK. Além do mais, no estado do Rio Grande do Sul, existem escassos estudos sobre a adubação em plantações de acácia-negra, como o de Maestri et al. (1987), testando diferentes doses de P e Mochiutti (2007), testando o efeito da aplicação de Ca e $\mathrm{Mg}$ e da adubação com $\mathrm{P}$ e K em diferentes tipos de solo. Esses autores verificaram que o crescimento a campo da Acacia mearnsii é diferenciado em função da quantidade e composição mineral da adubação utilizada no plantio. Objetivou-se, com o presente estudo, avaliar o crescimento da acácia-negra, em resposta a aplicação de nitrogênio, fósforo e potássio, após seis anos da implantação do povoamento.

\section{MATERIAL E MÉTODOS}

\subsection{Caracterização da área de estudo}

A área experimental foi instalada no distrito de Capão Comprido localizado no município de Butiá - RS, em área pertencente à empresa SETA S.A., estando localizado nas seguintes coordenadas geográficas: latitude $30^{\circ} 12^{\prime}$ 50,9" Sul e longitude $51^{\circ} 56^{\prime} 21,0^{\prime \prime}$ Oeste de Greenwich, a uma altitude média de $35 \mathrm{~m}$ acima do nível do mar.

O clima dominante da região, segundo Köppen é do tipo Cfa, subtropical. A temperatura média do mês de janeiro é de $24^{\circ} \mathrm{C}$, temperatura média do mês de julho é de $13^{\circ} \mathrm{C}$ e a temperatura média anual fica na faixa de $18-19^{\circ} \mathrm{C}$, com temperatura média máxima no ano de $24^{\circ} \mathrm{C}$ e mínima de $14^{\circ} \mathrm{C}$. A precipitação pluviométrica anual é $1.400 \mathrm{~mm}$ (MORENO, 1961).

Os atributos químicos do solo na área experimental antes da implantação do experimento podem ser visualizados na Tabela 1. Com base no Manual de Adubação e Calagem (SOCIEDADE BRASILEIRA DE CIÊNCIA DO SOLO, 2004), o teor de matéria orgânica $\left(<25 \mathrm{~g} \mathrm{~kg}^{-1}\right), \mathrm{pH}(\leq 5,0)$ e saturação por bases $(<45 \%)$ são considerados muito baixos; os teores de $\mathrm{P}\left(4,1-8,0 \mathrm{mg} \mathrm{dm}^{-3}\right)$,

Tabela 1 - Atributos do solo na área experimental, antes da instalação do experimento, no município de Butiá-RS.

Table 1 - Soil proprieties in experimental area, before the settlement of the experiment, in Butiá-RS.

\begin{tabular}{|c|c|c|c|c|c|c|c|c|c|c|c|c|c|}
\hline \multirow{2}{*}{$\begin{array}{l}\text { Prof. } \\
(\mathrm{cm})\end{array}$} & Arg. & M.O. & $\mathrm{C}_{\text {org. }}$ & $\mathrm{N}$ & \multirow{2}{*}{$\mathrm{C} / \mathrm{N}$} & $\mathrm{pH}$ & $\mathrm{K}$ & $\mathrm{P}$ & $\mathrm{Al}$ & $\mathrm{Ca}$ & $\mathrm{Mg}$ & \multicolumn{2}{|c|}{ Sat. (\%) } \\
\hline & \multicolumn{4}{|c|}{$\mathrm{g} \mathrm{kg}^{-1}$} & & $\mathrm{H}_{2} \mathrm{O}$ & \multicolumn{2}{|c|}{$\mathrm{mg} \mathrm{dm}^{-3}$} & \multicolumn{3}{|c|}{$\mathrm{cmol}_{\mathrm{c}} \mathrm{dm}^{-3}$} & $\mathrm{~V}$ & $\mathrm{~m}$ \\
\hline $0-20$ & 306 & 19 & 11,6 & 1,2 & 9,6 & 3,9 & 63,7 & 4,3 & 2,1 & 0,4 & 0,1 & 9,5 & 72,3 \\
\hline
\end{tabular}

A extração do $\mathrm{P}$ e $\mathrm{K}$ foi realizado com solução Mehlich 1, já o $\mathrm{Ca}, \mathrm{Mg}$ e o $\mathrm{Al}^{+3}$, em solução de $\mathrm{KCl}\left(1 \mathrm{~mol} \mathrm{~L}^{-1}\right)$.

Cerne, Lavras, v. 19, n. 1, p. 51-58, jan./mar. 2013 
$\mathrm{Ca} \mathrm{e} \mathrm{Mg}\left(\leq 2,0 \mathrm{e} \leq 0,45 \mathrm{cmol}_{c} \mathrm{dm}^{-3}\right.$, respectivamente) ) são considerados baixos; e os teores de $\mathrm{K}\left(61-120 \mathrm{mg} \mathrm{dm}^{-3}\right) \mathrm{e}$ saturação por alumínio (> 20\%) são considerados altos. Isso demonstra a baixa fertilidade natural do solo da área experimental. O solo da área em estudo é um Argissolo Vermelho-Amarelo distrófico típico, textura franco argilosa e relevo ondulado (STRECK et al., 2008).

\subsection{Descrição do experimento}

$\mathrm{O}$ experimento consiste em um delineamento de blocos completos ao acaso (três repetições) com a combinação de três doses de $\mathrm{N}\left(0,20 \mathrm{e} 40 \mathrm{~kg} \mathrm{ha}^{-1}\right.$, utilizandose uréia $(45 \%$ de $\mathrm{N})$ como fonte de $\mathrm{N})$, três doses de $\mathrm{P}(0,50$ e $100 \mathrm{~kg} \mathrm{ha}^{-1}$, utilizando-se superfosfato triplo $\left(46 \% \mathrm{de}_{2} \mathrm{O}_{5}\right)$ como fonte de $\mathrm{P})$ e três doses de $\mathrm{K}\left(0,25\right.$ e $50 \mathrm{~kg} \mathrm{ha}^{-1}$, utilizando-se cloreto de potássio $\left(60 \%\right.$ de $\left.\mathrm{K}_{2} \mathrm{O}\right)$ como fonte de $\mathrm{K}$ ). Cada uma das unidades amostrais possui dimensões de $18 \mathrm{~m}$ x $24 \mathrm{~m}$, com uma área total por parcela de $432 \mathrm{~m}^{2}$. As plantas se encontram arranjadas num espaçamento de $3,0 \mathrm{~m}$ x $1,33 \mathrm{~m}$, totalizando 108 plantas por unidade amostral, no momentoda instalação do experimento. O plantio foi realizado em julho de 2001, após o preparo de solo, que consistiu na eliminação das ervas daninhas na linha de plantio e subsolagem com uma haste até $45 \mathrm{~cm}$ de profundidade. $\mathrm{Na}$ Tabela 2, estão descritos os 27 tratamentos utilizados no experimento com as doses de cada nutriente.

Tabela 2 - Tratamentos avaliados no experimento de adubação mineral (NPK) em acácia-negra, Butiá - RS.

Table 2 - Treatments in the black-wattle mineral fertilizer experiment (NPK), Butiá-RS.

\begin{tabular}{|c|c|c|c|c|c|c|c|c|}
\hline \multirow{3}{*}{ Trat. } & \multicolumn{6}{|c|}{ Nutrientes } & \multirow{2}{*}{\multicolumn{2}{|c|}{ Total }} \\
\hline & \multicolumn{2}{|c|}{$\mathrm{N}$} & \multicolumn{2}{|c|}{$\mathrm{P}$} & \multicolumn{2}{|c|}{$\mathrm{K}$} & & \\
\hline & $\mathrm{kg} \mathrm{ha}^{-1}$ & g planta $^{-1}$ & $\mathrm{~kg} \mathrm{ha}^{-1}$ & g planta $^{-1}$ & $\mathrm{~kg} \mathrm{ha}^{-1}$ & g planta $^{-1}$ & $\mathrm{~kg} \mathrm{ha}^{-1}$ & g planta $^{-1}$ \\
\hline 1 & 0 & 0 & 0 & 0 & 0 & 0 & 0 & 0 \\
\hline 2 & 0 & 0 & 0 & 0 & 25 & 10 & 25 & 10 \\
\hline 3 & 0 & 0 & 0 & 0 & 50 & 20 & 50 & 20 \\
\hline 4 & 0 & 0 & 50 & 20 & 0 & 0 & 50 & 20 \\
\hline 5 & 0 & 0 & 50 & 20 & 25 & 10 & 75 & 30 \\
\hline 6 & 0 & 0 & 50 & 20 & 50 & 20 & 100 & 40 \\
\hline 7 & 0 & 0 & 100 & 40 & 0 & 0 & 100 & 40 \\
\hline 8 & 0 & 0 & 100 & 40 & 25 & 10 & 125 & 50 \\
\hline 9 & 0 & 0 & 100 & 40 & 50 & 20 & 150 & 60 \\
\hline 10 & 20 & 8 & 0 & 0 & 0 & 0 & 20 & 8 \\
\hline 11 & 20 & 8 & 0 & 0 & 25 & 10 & 45 & 18 \\
\hline 12 & 20 & 8 & 0 & 0 & 50 & 20 & 70 & 28 \\
\hline 13 & 20 & 8 & 50 & 20 & 0 & 0 & 70 & 28 \\
\hline 14 & 20 & 8 & 50 & 20 & 25 & 10 & 95 & 38 \\
\hline 15 & 20 & 8 & 50 & 20 & 50 & 20 & 120 & 48 \\
\hline 16 & 20 & 8 & 100 & 40 & 0 & 0 & 120 & 48 \\
\hline 17 & 20 & 8 & 100 & 40 & 25 & 10 & 145 & 58 \\
\hline 18 & 20 & 8 & 100 & 40 & 50 & 20 & 170 & 68 \\
\hline 19 & 40 & 16 & 0 & 0 & 0 & 0 & 40 & 16 \\
\hline 20 & 40 & 16 & 0 & 0 & 25 & 10 & 65 & 26 \\
\hline 21 & 40 & 16 & 0 & 0 & 50 & 20 & 90 & 36 \\
\hline 22 & 40 & 16 & 50 & 20 & 0 & 0 & 90 & 36 \\
\hline 23 & 40 & 16 & 50 & 20 & 25 & 10 & 115 & 46 \\
\hline 24 & 40 & 16 & 50 & 20 & 50 & 20 & 140 & 56 \\
\hline 25 & 40 & 16 & 100 & 40 & 0 & 0 & 140 & 56 \\
\hline 26 & 40 & 16 & 100 & 40 & 25 & 10 & 165 & 66 \\
\hline 27 & 40 & 16 & 100 & 40 & 50 & 20 & 190 & 76 \\
\hline
\end{tabular}

Cerne, Lavras, v. 19, n. 1, p. 51-58, jan./mar. 2013 
Uma vez no campo, inicialmente as mudas foram coroadas até um raio de $30 \mathrm{~cm}$ e após a abertura de um sulco circular a $20 \mathrm{~cm}$ de distância da planta o adubo foi aplicado com incorporação. Para avaliação, aos seis anos de idade das plantas, foram mensuradas apenas as árvores da área útil da parcela, considerando bordadura dupla. Avaliou-se a altura total das árvores (m), com o auxilio do hipsômetro Vertex e diâmetro à altura do peito $(\mathrm{cm})$, com uso de fita diamétrica. Com base nessas duas vaiáveis e a aplicação de um fator de forma $(0,55)$ obteve-se o volume de madeira com casca $\left(\mathrm{m}^{3} \mathrm{ha}^{-1}\right)$.

No estudo dos efeitos dos fatores e seus respectivos níveis sobre as variáveis avaliadas no experimento (altura total, diâmetro a altura do peito e volume com casca), foi ajustada uma superfície de resposta com nível de significância de $5 \%$.

O modelo matemático utilizado foi:

$\mathrm{Y}=\beta_{0}+\beta_{1} \mathrm{x}_{1}+\beta_{11} \mathrm{x}_{1}{ }_{1}+\beta_{2} \mathrm{x}_{2}+\beta_{22} \mathrm{x}_{2}{ }_{2}+\beta_{3} \mathrm{x}_{3}+\beta_{33} \mathrm{x}_{3}{ }_{3}+$ $\beta_{12} x_{1} x_{2}+\beta_{13} x_{1} x_{2}+\beta_{13} x_{1} x_{3}+\beta_{23} x_{2} x_{3}+\varepsilon$, em que: $\mathrm{x}_{1}=$ dose de $\mathrm{N}$ variando de 0 a $40 \mathrm{~kg} \mathrm{ha}^{-1}$;

$\mathrm{x}_{2}=$ dose de $\mathrm{P}$ variando de 0 a $100 \mathrm{~kg} \mathrm{ha}^{-1}$;

$\mathrm{x}_{3}=$ dose de $\mathrm{K}$ variando de 0 a $50 \mathrm{~kg} \mathrm{ha}^{-1}$;

$\beta_{0}, \beta_{1}, \beta_{11}, \beta_{2}, \beta_{22}, \beta_{3}, \beta_{33}, \beta_{12}, \beta_{13}, \beta_{13}$ e $\beta_{23}=$ parâmetros da equação;

$\varepsilon=$ vetor dos desvios das médias estimadas em relação ao modelo.

As doses de Máxima Eficiência Técnica (MET) foram estimadas algebricamente, a partir do ajuste da superfície de resposta (equação polinomial de segundo grau). Em razão da mortalidade de alguns indivíduos nas parcelas experimentais, aos seis anos de idade, verificouse a necessidade de ajuste das médias das variáveis (DAP, Altura e Volume) com base na covariável número de plantas. Todas as análises estatísticas foram realizadas com o auxílio do pacote estatístico SAS (STATISTICAL ANALYSIS SYSTEM INSTITUTE - SAS INSTITUTE, 1999).

\section{RESULTADOS E DISCUSSÃO}

Na Tabela 3, são apresentados o diâmetro médio à altura do peito (DAP), a altura média das árvores, a percentagem de sobrevivência e o volume com casca, por tratamento, aos 72 meses de idade.

Em plantações de acácia-negra a mortalidade reduz, significativamente, a densidade de árvores no final da rotação, que apresenta correlação negativa com a produção de madeira (MOCHIUTTI, 2007), em razão
Tabela 3 - Variáveis dendrométricas de plantas de acácia-negra (Acacia mearnsii De Wild.) aos 6 anos de idade em função da adição de diferentes doses de NPK.

Table 3 - Dendrometric variables of black-wattle plants (Acacia mearnsii De Wild.) at 6 years-age, depending on the addition of different doses of NPK.

\begin{tabular}{lcccc}
\hline $\begin{array}{l}\text { Doses } \\
\text { N-P-K }\left(\mathrm{kg} \mathrm{ha}^{-1}\right)\end{array}$ & $\begin{array}{c}\text { DAP } \\
(\mathrm{cm})\end{array}$ & $\begin{array}{c}\text { Altura } \\
(\mathrm{m})\end{array}$ & $\begin{array}{c}\text { Sobrev. } \\
(\%)\end{array}$ & $\begin{array}{c}\text { Volume } \\
\left(\mathrm{m}^{3} \mathrm{ha}^{-1}\right)\end{array}$ \\
\hline $0-0-0$ & 10,7 & 15,7 & 67 & 161,0 \\
$0-0-25$ & 11,7 & 16,3 & 71 & 188,0 \\
$0-0-50$ & 10,9 & 15,1 & 67 & 152,1 \\
$0-50-0$ & 11,3 & 16,6 & 74 & 183,6 \\
$0-50-25$ & 11,9 & 16,9 & 78 & 216,8 \\
$0-50-50$ & 11,7 & 16,2 & 71 & 183,6 \\
$0-100-0$ & 12,0 & 16,7 & 72 & 205,9 \\
$0-100-25$ & 11,9 & 16,5 & 69 & 198,5 \\
$0-100-50$ & 12,4 & 16,4 & 74 & 218,1 \\
$20-0-0$ & 10,2 & 14,5 & 71 & 133,5 \\
$20-0-25$ & 10,4 & 15,3 & 77 & 153,1 \\
$20-0-50$ & 10,4 & 15,1 & 77 & 152,2 \\
$20-50-0$ & 13,0 & 18,2 & 63 & 219,3 \\
$20-50-25$ & 12,4 & 17,5 & 75 & 231,0 \\
$20-50-50$ & 12,5 & 17,4 & 71 & 235,7 \\
$20-100-0$ & 12,9 & 16,9 & 65 & 225,5 \\
$20-100-25$ & 12,6 & 18,1 & 70 & 230,2 \\
$20-100-50$ & 12,8 & 17,9 & 74 & 247,3 \\
$40-0-0$ & 11,8 & 17,2 & 70 & 195,4 \\
$40-0-25$ & 11,9 & 16,7 & 63 & 176,2 \\
$40-0-50$ & 11,6 & 16,6 & 62 & 161,5 \\
$40-50-0$ & 12,0 & 17,1 & 67 & 192,2 \\
$40-50-25$ & 12,2 & 16,6 & 72 & 212,8 \\
$40-50-50$ & 12,5 & 17,2 & 67 & 208,0 \\
$40-100-0$ & 12,3 & 18,1 & 69 & 220,7 \\
$40-100-25$ & 12,1 & 17,0 & 69 & 208,2 \\
$40-100-50$ & 12,3 & 16,5 & 67 & 190,5 \\
\hline & & & & \\
\hline & 15,4 &
\end{tabular}

disso, optou-se por realizar um ajuste das médias dos tratamentos em função da covariável (número de plantas). Essa análise de covariância tem como objetivo controlar o erro, aumentar a precisão da análise de dados e ajustar as médias dos tratamentos em função da covariável.

\section{Cerne, Lavras, v. 19, n. 1, p. 51-58, jan./mar. 2013}


Um dos requisitos indispensável para a realização da análise de variância no ajuste de médias de tratamentos é a não correlação da covariável (número de plantas) com os tratamentos. Dessa forma, pela não significância da análise de variância, a covariável tem comportamento independente dos tratamentos, portanto, sendo conveniente estudar as diferenças entre as médias de y (DAP, altura e volume) ajustadas para as médias de $\mathrm{x}$ (covariável). Por meio da análise de variância, verificou-se que o ajuste das médias, com base na covariável número de plantas, é significativo somente para o volume de madeira com casca.

Após ser realizada a análise da covariância e realizado os ajustes nas variáveis foi efetuada a análise da variância para as variáveis analisadas. Verificou-se que para as variáveis dependentes em estudo (altura total, diâmetro a altura do peito e volume com casca) não houve interação entre as doses de N e K; P e K (p > 0,05). Já, as interações dos fatores $\mathrm{N}$ e $\mathrm{P}$ foram significativas $(\mathrm{p}<0,05)$ no crescimento da acácia-negra aos seis anos de idade.

Os resultados deste trabalho demonstraram que o $\mathrm{P}$ foi o nutriente mais importante para o crescimento das plantas de acácia-negra. Essa resposta a fertilização mineral com fósforo, decorre do baixo teor disponível desse elemento no solo $\left(4,3 \mathrm{mg} \mathrm{dm}^{-3}\right) \mathrm{e}$, também, ao suprimento de cálcio via superfosfato triplo (12 a $14 \%$ do adubo mineral é composto por $\mathrm{Ca}$ ). As plantas de acácia-negra, provavelmente apresentaram respostas acentuadas à adição de cálcio via superfosfato triplo, visto que, o teor trocável desse elemento no solo estava abaixo do limite inferior de suprimento $\left(2,0 \mathrm{cmol} \mathrm{dm}_{c}^{-3}\right)$ e, além do mais, não foi realizada a correção da acidez do solo via aplicação de calcário.

A influência no crescimento da acácia-negra com a adição de fósforo via fertilizante mineral tem sido apontada por outros estudos. Respostas lineares a aplicação de doses de 0 a 486, $1 \mathrm{~kg} \mathrm{ha}^{-1}$ de superfosfato (8,3\% de P) sobre o DAP, altura, área basal e volume de madeira foram encontradas na África do Sul (HERBERT, 1991). No Rio Grande do Sul, Maestri et al. (1987) avaliaram diversas fontes e doses $\mathrm{P}$, encontrando efeito linear para superfosfato simples (doses de 0 a $750 \mathrm{~kg}$ ha $^{-1}$ ), termofosfato (doses de 0 a $950 \mathrm{~kg} \mathrm{ha}^{-1}$ ) e hiperfosfato (doses 0 a $1.150 \mathrm{~kg} \mathrm{ha}^{-1}$ ), e efeito quadrático para fosfato natural de Araxá (doses de 0 a $3.000 \mathrm{~kg} \mathrm{ha}^{-1}$ ).

O comportamento das variáveis dendrométricas analisadas em função da disponibilização de nutrientes $(\mathrm{N}, \mathrm{P}$ e K) e suas doses, geralmente, está associado às demandas nutricionais da cultura que, por sua vez, variam em função da espécie, fase de desenvolvimento, solo etc. Quanto ao nitrogênio, o seu efeito significativo sobre o crescimento de uma espécie leguminosa fixadora desse elemento, também foi verificado em estudo realizado por Pinkard (2003) com Acacia melanoxylon. Segundo o autor, o crescimento em altura foi superior com a aplicação de fósforo, já, o diâmetro a altura do peito, a aplicação tanto do fósforo como de nitrogênio ou a aplicação conjunta dos dois elementos, proporcionaram maior crescimento.

Já, o potássio, alguns estudos indicam que a demanda por esse elemento é menos expressiva nas fases iniciais dos povoamentos florestais, quando comparado ao nitrogênio e, principalmente, ao fósforo. Em estudo realizado por Tedesco (1999) foi verificado que combinação de N e P proporcionou aumento da altura e diâmetro de mudas de Acacia mearnsii, enquanto que o $\mathrm{K}$ não apresentou efeito e nem interação com $\mathrm{NeP}$ para as variáveis estudadas. Clauberg (2005) também não verificou efeito da adubação potássica no crescimento inicial a campo de Mimosa scabrella. Os teores de Pe K no solo eram, respectivamente, de 4,8 e $62 \mathrm{mg} \mathrm{dm}^{-3}$, para o primeiro estudo citadoe $1,1 \mathrm{e} 80 \mathrm{mg} \mathrm{dm}^{-3}$, para o segundo. Andrade et al. (2004) não observaram interação entre as doses de $\mathrm{P}$ e $\mathrm{K}$, no crescimento da acácia-negra aos 61 meses de idade, em solo com baixos teores desses nutrientes, mas obtiveram efeitos significativos com a aplicação de diferentes doses de P ou K.

Para a superfície de resposta estimada, determinaram-se os valores de $\mathrm{x}_{1}(\mathrm{~N}) \mathrm{e}_{2}(\mathrm{P})$ que determinam o valor de Y. Na Figura 1, pode ser observada a superfície de resposta da variável diâmetro a altura do peito, em função das diferentes doses de nitrogênio e fósforo, a qual apresenta um ponto de máximo em $40,4 \mathrm{~kg} \mathrm{ha}^{-1}$ de $\mathrm{Ne}$ 78,9 $\mathrm{kg} \mathrm{ha}^{-1}$ de $\mathrm{P}$, correspondendo à máxima eficiência técnica (MET) de 12,5 cm de diâmetro a altura do peito.

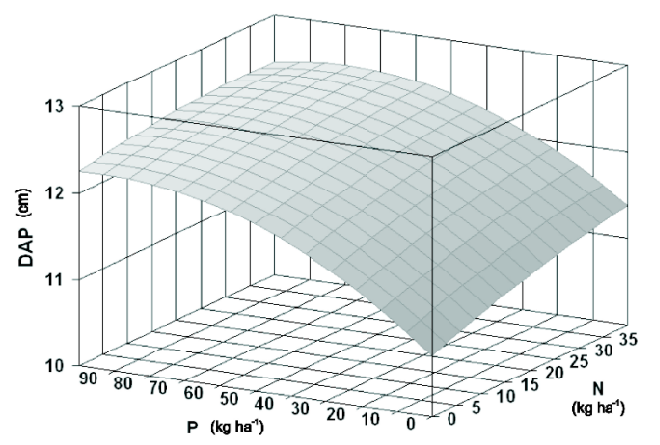

Figura 1 - Diâmetro a altura do peito de plantas de acácianegra aos seis anos de idade em função das doses de nitrogênio e fósforo. $y(D A P)=10,701543+0,024120 \mathrm{~N}+0,033537 \mathrm{P}-$ $0,000176 \mathrm{~N}^{2}-0,000119 \mathrm{NP}-0,000181 \mathrm{P}^{2}\left(\mathrm{R}^{2}=0,698\right)$.

Figure 1 -Diameter at breast height of black-wattle plants at six years-age depending on the nitrogen and phosphorus doses.

Cerne, Lavras, v. 19, n. 1, p. 51-58, jan./mar. 2013 
Entretanto, na Figura 2, pode ser observada a superfície de resposta da altura total, em função das diferentes doses de nitrogênio e fósforo, a qual apresenta um ponto de máximo em $48,0 \mathrm{~kg} \mathrm{ha}^{-1} \mathrm{de} \mathrm{N}$ e $69,5 \mathrm{~kg} \mathrm{ha}^{-1} \mathrm{de} \mathrm{P}$, correspondendo à máxima eficiência técnica de $17,5 \mathrm{~m}$.

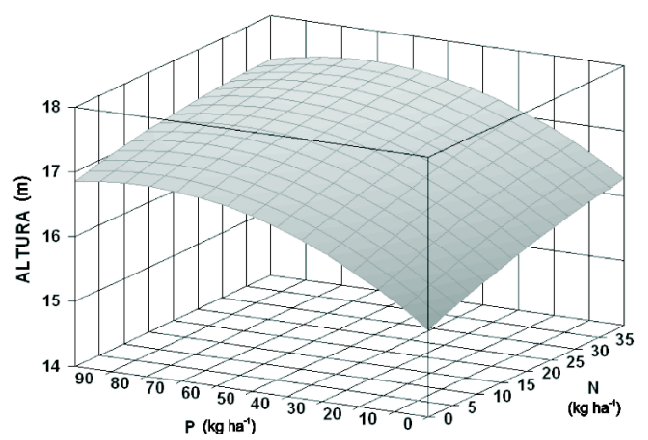

Figura 2 - Altura total de plantas de acácia-negra aos seis anos de idade em função das doses de nitrogênio e fósforo. y(altura) = $15,338889+0,033241 \mathrm{~N}+0,039519 \mathrm{P}-0,000250 \mathrm{~N}^{2}-$ $0,000117 N P-0,000244 P^{2}\left(R^{2}=0,647\right)$.

Figure 2 - Total height of black-wattle plants at six years-age depending on the nitrogen and phosphorus doses.

A superfície de resposta do volume de madeira (Figura 3), em função das diferentes doses de nitrogênio e fósforo, a qual apresenta um ponto máximo em $62,7 \mathrm{~kg} \mathrm{ha}^{-1}$ de $\mathrm{Ne} 74,0 \mathrm{~kg} \mathrm{ha}^{-1}$ de $\mathrm{P}$, correspondendo à máxima eficiência técnica de $226,3 \mathrm{~m}^{3} \mathrm{ha}^{-1}$.

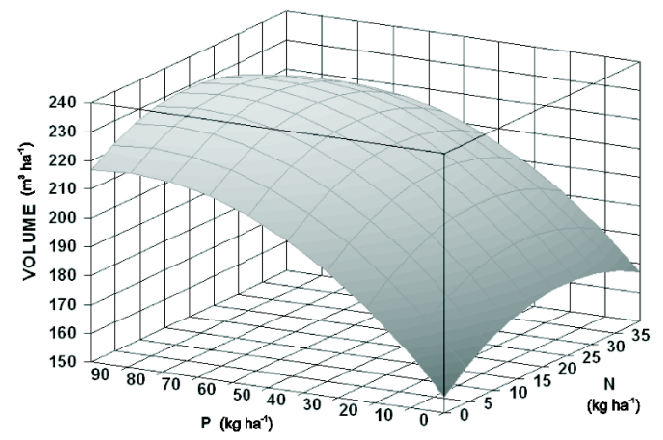

Figura 3 - Volume com casca de plantas de acácia-negra aos seis anos de idade em função das doses de nitrogênio e fósforo. $\mathrm{y}($ volume $)=155,763160+1,608861 \mathrm{~N}+1,377004 \mathrm{P}-$ $0,033132 \mathrm{~N}^{2}-0,003147 \mathrm{NP}-0,007679 \mathrm{P}^{2}\left(\mathrm{R}^{2}=0,771\right)$.

Figure 3 - Volume with bark of black-wattle plants at six yearsage depending on the nitrogen and phosphorus doses.
Para Mochiutti (2007), a produção máxima do volume de madeira com casca de acácia-negra foi com a dose de $\mathrm{P}$ $\left(67,0 \mathrm{~kg} \mathrm{ha}^{-1}\right.$ de $\left.\mathrm{P}_{2} \mathrm{O}_{5}\right)$ juntamente com $20,6 \mathrm{~kg} \mathrm{ha}^{-1}$ de $\mathrm{K}_{2} \mathrm{O}$. A produtividade obtida para essas doses representaram um aumento de $36,2 \%$ em relação ao tratamento sem adubação.

A resposta mais acentuada em crescimento em altura da Acacia melanoxylon foi verificada com aplicação de fósforo, já, para o diâmetro, foi obtido com nitrogênio ou da combinação de N e P (PINKARD, 2003). Cobbina (1991) obteve aumento no crescimento inicial de Leucaena leucoceohala por meio da adubação nitrogenada e aplicação de fósforo.

\section{CONCLUSÕES}

Para a obtenção do máximo crescimento da acácianegra, na condição edafoclimática estudada, deve-se utilizar a dose máxima de nitrogênio $\left(40,0 \mathrm{~kg} \mathrm{ha}^{-1} \mathrm{de} \mathrm{N}\right)$ e $78,9 \mathrm{~kg} \mathrm{ha}^{-1}$ de fósforo, não sendo necessária a adição de potássio via fertilizantes.

\section{AGRADECIMENTOS}

À empresa SETAS.A., pela disponibilização da área para instalação da pesquisa e o apoio logístico-financeiro e aos três revisores anônimos, pelas sugestões e pelos questionamentos, os quais foram muito bem aceitos, melhorando a qualidade deste artigo.

\section{REFERÊNCIAS}

ANDRADE, G. de C.; BELLOTE, A. F. J.; FERREIRA, C. A.; SILVA, H. D. da. Adubação fosfatada e potássica em plantios de Acacia mearnssi De Wild. (Acácia-negra). Boletim de Pesquisa Florestal, Colombo, n. 48, p. 65-74, jan./jun. 2004.

BARROS, N. F.; NEVES, J. C. L.; NOVAIS, R. F. Mineral fertilizer recommendations for eucalypt plantations. In: GONÇALVES, J. L. M.; BENEDETTI, V. (Ed.). Forest nutrition and fertilization. Piracicaba: IPEF, 2004. p. 269284.

BARROS, N. F.; NOVAIS, R. F. Relação solo-eucalipto. Viçosa, MG: Folha de Viçosa, 1990. 330 p.

CALDEIRA, M. V. W.; RONDON NETO, R. M.; SCHUMACHER, M. V. Acúmulo e exportação de micronutrientes em um povoamento e acácia-negra (Acacia mearnsii De Wild.) procedência Bodalla - Austrália. Revista Floresta, Curitiba, v. 33, n. 1, p. 73-78, 2003.

Cerne, Lavras, v. 19, n. 1, p. 51-58, jan./mar. 2013 
CALDEIRA, M. V. W.; SCHUMACHER, M. V.; SANTOS, L. M. dos; TEDESCO, N.; PEREIRA, S. C. Estimativa do conteúdo de nutrientes em um povoamento jovem de Acacia mearnsii De Wild. estabelecido na região sul do Brasil.

Revista Floresta, Curitiba, v. 29, n. 1/2, p. 53-65, 1999.

CLAUBERG, D. Adubação N-P-K em Mimosa scabrella (Benth.). 2005. 62 p. Dissertação (Mestrado em Engenharia Ambiental) - Universidade Regional de Blumenau, Blumenau, 2005.

COBBINA, J. Nitrogen and phosphorus fertilization promotes rapid initial growth of Leucaena on alfisol. Communications in Soil Science and Plant Analysis, New York, v. 22, n. 1/2, p. 1-9, Jan. 1991.

DALLAGO, J. S. Utilização da cinza de biomassa de caldeira como fonte de nutrientes no crescimento de plantas de acácia-negra (Acacia mearnsii De Wild.). 2000. 64 p. Dissertação (Mestrado em Engenharia Florestal) Universidade Federal de Santa Maria, Santa Maria, 2000.

FORRESTER, D. I.; COWIE, A. L.; BAUHUS, J.; WOOD, J. T.; FORRESTER, R. I. Eûects of changing the supply of nitrogen and phosphorus on growth and interactions between Eucalyptus globulus and Acacia mearnsii in a pot trial. Plant and Soil, The Hague, v. 280, n. 1/2, p. 267-277, Feb. 2006.

GONÇALVES, J. L. M. Recomendações de adubação para Eucalyptus, Pinus e espécies típicas da Mata Atlântica. Documentos Florestais, Piracicaba, n. 15, p. 1-23, jan./dez. 1995.

GONÇALVES, J. L. M.; BARROS, N. F. Improvement of site productivity for short-rotation plantations in Brazil. Bosque, Valdivia, v. 20, n. 1, p. 89-106, 1999.

GONÇALVES, J. L. M.; STAPE, J. L.; LACLAU, J. P.; BOUILLET , J. P.; RANGER, J. Assessing the effects of early silvicultural management on long-term site productivity of fast-growing eucalypt plantations: the Brazilian experience. Southern Forests, Grahamstown, v. 70, n. 2, p. 105-118, Aug. 2008.

HERBERT, M. A. Fertilization of trees at planting. In: MACLENNAM, L. (Ed.). Annual research report. Pietermaritzburg: Institute for Commercial Forestry Research, 1991. p. 81-91.
MAESTRI, R.; GRAÇA, L. R.; SIMÕES, J. W.; FREITAS, A. J. P. Análise da adubação fosfatada na produção física e econômica da acácia-negra. Boletim de Pesquisa Florestal, Colombo, n. 14, p. 39-53, jun. 1987.

MOCHIUTTI, S. Produtividade e sustentabilidade de plantações de acácia-negra (Acacia mearnsii De Wild.) no Rio Grande do Sul. 2007. 270 p. Tese (Doutorado em Ciências Florestais) - Universidade Federal do Paraná, Curitiba, 2007.

MOREnO, J. A. Clima do Rio Grande do Sul. Porto Alegre: Secretaria da Agricultura, 1961. 42 p.

PARDOS, M.; ROYO, A.; PARDOS, J. A. Growth, nutrient, water relations, and gas exchange in a holm oak plantation in response to irrigation and fertilization. New Forests, Dordrecht, v. 30, n. 1, p. 75-94, July 2005.

PINKARD, E. A. Growth and physiological responses of Blackwood (Acacia melanoxylon) growing with a Pinus radiata nurse crop following applications of nitrogen and phosphorus. Trees, Berlin, v. 17, n. 4, p. 325-335, July 2003.

RYAN, M. G. Factors controlling Eucalyptus productivity: how water availability and stand structure alter production and carbon allocation. Forest Ecology Management, Amsterdam, v. 259 , n. 9 , p. 1695-1703, 2010.

SCHÖNAU, A. P. G. A site evoluation study in Black Wattle (Acacia mearnsii De Wild.). Annale van die Universiteit von Stellenbosch, Stellenbosh, v. 44, n. 2, p. 79-214, 1969.

SIMON, A. A. A cadeia produtiva da acácia-negra, aspectos econômicos, sociais e ambientais. In: REFLORESTAMENTO E RECUPERAÇÃO AMBIENTAL. AMBIENTE E TECNOLOGIA: O DESENVOLVIMENTO SUSTENTÁVEL EM FOCO, 3., 2005, Lajeado. Anais... Lajeado: UNIVATES, 2005. p. $149-166$.

SMETHURST, P. J. Forest fertilization: trends in knowledge and practice compared to agriculture. Plant and Soil, The Hague, v. 335, n. 1/2, p. 83-100, 2010.

SMETHURST, P. J. Soil solution and other soil analyses as indicators of nutrient supply: a review. Forest Ecology Management, Amsterdam, v. 138, n. 1/3, p. 397-411, Nov. 2000.

Cerne, Lavras, v. 19, n. 1, p. 51-58, jan./mar. 2013 
SOCIEDADE BRASILEIRA DE CIÊNCIA DO SOLO. Comissão de Química e Fertilidade do Solo. Manual de adubação e calagem para os estados do Rio Grande do Sul e de Santa Catarina. 10. ed. Porto Alegre, 2004. 400 p.

STATISTICAL ANALYSIS SYSTEM INSTITUTE. SAS/

STAT user's guide. Version 8. Cary, 1999.

STRECK, E. V.; KÄMPF, N.; DALMOLIN, R. S. D.; KLAMT, E.; NASCIMENTO, P. C.; SCHNEIDER, P.; GIASSON, E.; PINTO, L. F. S. Solos do Rio Grande do Sul. 2. ed. Porto Alegre: EMATER/RS, 2008. 222 p.
TEDESCO, N. Produção de mudas de acácia-negra (Acacia mearnsii De Wild.) adubadas com N-P-K. 1999. 71 p. Dissertação (Mestrado em Engenharia Florestal) - Universidade Federal de Santa Maria, Santa Maria, 1999.

VOGEL, H. L. M. Crescimento inicial de Pinus taeda $\mathbf{L}$. relacionado a doses de $\mathrm{N}, \mathrm{P}$ e $\mathrm{K}$, e sua diagnose nutricional pelos dris. 2002. 83 p. Dissertação (Mestrado em Engenharia Florestal) - Universidade Federal de Santa Maria, Santa Maria, 2002. 